\title{
Traceable radiation thermometer calibration to ITS-90 above the silver point at the National Physical Laboratory
}

\author{
David Lowe and Graham Machin \\ National Physical Laboratory, Hampton Road, Teddington, UK
}

\begin{abstract}
Résumé. Le National Physical Laboratory est accrédité par le United Kingdom Accreditation Service (UKAS) pour fournir ITS-90 étalonnage de la température des thermomètres à radiation avec une meilleure mesure la capacité incertitude de $0,05 \%(k=2)$ de la température de $1000^{\circ} \mathrm{C}$ à $3000^{\circ} \mathrm{C}$. Pour être en mesure de fournir ce niveau d'incertitude comme un service de routine nécessite très faibles incertitudes dans l'équipement utilisé pour réaliser et diffuser l'échelle. Tous les certificats délivrés pour l'étalonnage de la température sont fait en termes de l'ITS-90, mais cela peut être réalisé de deux manières très différentes sur le plan conceptuel. Tout d'abord, par comparaison avec un thermomètre à rayonnement standard qui a lui-même été étalonné directement conformément à l'ITS-90 définition, et à médiation par l'utilisation d'un corps noir à température variable; ou, d'autre part, par la mesure de sources à haute température fixe le point de corps noirs qui ont a priori affectée ITS-90 températures. Ce document décrit l'équipement et les procédés utilisés au NPL pour étalonner les pyromètres à ITS-90 ci-dessus le point d'argent avec les incertitudes les plus bas de ces deux routes.
\end{abstract}

\section{Introduction}

National Metrology Institutes (NMIs), especially those who are signatories to the CIPM Mutual Recognition Arrangement, have a confirmed equivalence of measurement capability based on intercomparison between institutes. The measurement standards at each NMI then form the base of a system of traceability for measurements within each country. This system promotes standardisation and reduces trade barriers. Within the UK, the NMI is the National Physical Laboratory (NPL). NPL maintains the national standards to which all reliable measurements are ultimately traceable. This traceability is assured by the United Kingdom Accreditation Service (UKAS), the sole national accreditation body recognised by the UK government to assess, against internationally agreed standards, organisations that provide certification, testing, inspection and calibration services. For temperature measurement above the freezing point of silver at $961.78^{\circ} \mathrm{C}$ NPL is accredited by UKAS to calibrate radiation thermometers with a calibration and measurement capability (CMC), expressed as an expanded uncertainty $(k=2)$, as low as $0.05 \%$ of temperature in Celsius. All temperature calibration is in terms of the International Temperature Scale of 1990 (ITS-90) [1], but instruments can be calibrated by two distinct methods: a) By using a radiation thermometer standard calibrated directly in accordance with the ITS-90 definition. This calibration is transferred to a customer's radiation thermometer via a variable temperature blackbody source. This is the most generally applicable method. Any customer radiation thermometer with a field-of-view less than $20 \mathrm{~mm}$ at a working distance greater than $500 \mathrm{~mm}$ can be calibrated at NPL in this way. The calibration uncertainty is generally limited by the performance of the test thermometer, and this usually means the final uncertainty is considerably greater than $0.05 \%$ of Celsius temperature.

b) By measurement of high temperature fixed point (HTFPs) [2] blackbody sources with a priori assigned ITS-90 temperatures. This is a more specialised service limited to radiation thermometers with a target size of less than $1.5 \mathrm{~mm}$ and which have a sufficiently narrow spectral response band to approximate their output to the Planck radiation law. The advantage of this approach is that even a fairly simple radiation thermometer can be calibrated with an uncertainty approaching the best measurement capability of $0.05 \%$ of Celsius temperature [3].

Both methods require a highly linear reference radiation thermometer to assign the temperature to either the variable temperature or HTFP sources and the capability of determining its spectral response. However, it is expected that in the future the mise en pratique of the 
definition of the kelvin $(M e P-K)$ [4,5] will provide defined melting temperature values and uncertainties for HTFPs as a formal approximation to the kelvin, and remove both these requirements, since thermometers can be calibrated directly against the HTFPs. At that time, the second method will become much simpler to realise, and much of the equipment and procedure for realising the ITS-90 could become redundant. This paper describes the methodology required for both routes.

\section{Scale Realisation}

A primary scale requires that it is established in terms of the definition of the unit. For ITS-90 this means using Planck's law to give the ratio of spectral radiance $L_{k}$. emitted by an object of unknown temperature, $T_{90}$, to that emitted by an object at a specified reference temperature $T_{90}(X)[1]$

$$
\frac{L_{\lambda}\left(T_{90}\right)}{L_{\lambda}\left[T_{90}(X)\right]}=\frac{\exp \left(c_{2}\left[\lambda T_{90}(X)\right]^{-1}\right)-1}{\exp \left(c_{2}\left[\lambda T_{90}\right]^{-1}\right)-1}
$$

where $T_{90}(\mathrm{X})$ is the freezing temperature of either silver, gold or copper and $c_{2}$ is the second radiation constant. At NPL ultimate traceability is to the freezing temperature of copper using a fixed-point blackbody cell in a three-zone furnace, which itself has been validated by direct or indirect comparison to other NMIs [6]. The copper point cell has a $3 \mathrm{~mm}$ aperture with an emissivity $0.99998 \pm$ 0.00001 and contains $345 \mathrm{~g}$ of $99.9999 \%$ pure copper.

\subsection{By comparison to a calibrated standard}

In brief, at NPL a KE- Technologie GmbH LP3 radiation thermometer [7] fitted with an apochromate objective lens [8] is used as the reference radiation thermometer and hence the foundation of the NPL ITS-90 realisation above the silver point. It operates at independent wavelengths of $650 \mathrm{~nm}$ and $900 \mathrm{~nm}$, has a very well defined target size of less than $1 \mathrm{~mm}$ with a very low size-of-source effect (SSE), and is linear to better than 5 parts in $10^{4}$ across signals that vary by 4 orders of magnitude. The LP3 is characterised for these parameters and then calibrated using the copper point reference blackbody. The LP3 is then used to assign a temperature to a Thermogauge dual blackbody radiance source, which can be used as a radiance source up to $3000{ }^{\circ} \mathrm{C}$ for calibration of radiation thermometers.

\subsubsection{Determination of the LP3 Spectral response}

A monochromator and a tungsten ribbon lamp (TRL) as a source are used for this measurement. The TRL, which can be interchanged with a spectral line source, illuminates the monochromator input slit. The LP3 is focussed on the output slit. The LP3 can be set with no interference filter in its optical path. The monochromator is scanned with no filter while the LP3 output is recorded, first with the line source and then with the TRL. The scan with the TRL is repeated with the interference filter in position. The relative spectral response (which here includes the detector response) is simply the ratio of the LP3 filtered and open signals. The line source allows the wavelength correction for the monochromator to be determined. For a stable narrow band filter the uncertainty in this correction and reliable estimation of the filter's out-of-band transmission are the dominant contributions to the uncertainty; and wavelength calibration is the dominant contributor to the overall uncertainty budget. The overall instrument response depends both on the filter and detector response and also the Planck function that describes the radiance of the measurand. The spectral response therefore changes with the temperature of the measurand and this dependence is accounted for by use of an effective wavelength

$$
\lambda_{\text {eff }}=A+B / T
$$

where $A$ is related to the central wavelength and $B$ describes the change in apparent wavelength. The wavelength used in solving (1) is the mean of the effective wavelengths at $T_{90}(\mathrm{X})$ and $T_{90}, \overline{\lambda_{\text {eff }}}$ which requires iteration to successively closer approximations to $\overline{\lambda_{e f f}}$. A full spectral response calibration of the LP3 is carried out every two years.

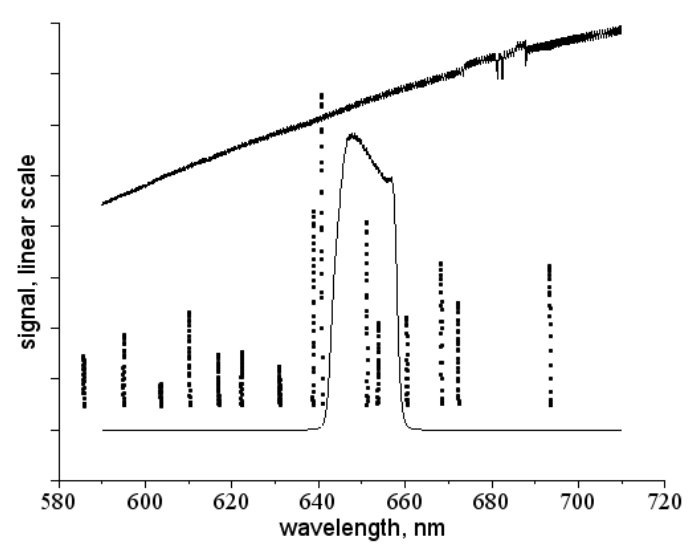

Figure 1. The filter in, filter out and line source spectra for the LP3 $650 \mathrm{~nm}$ filter allow its effective wavelength to be determined with an uncertainty of $0.065 \mathrm{~nm}$

\subsubsection{Size-of-source effect}

The next largest uncertainty arises from the influence of signal originating from outside the LP3's nominal fieldof-view but which is scattered such that it reaches the detector and causes an additional signal, characterised by the SSE. As a consequence of the SSE the signal will increase with the size of the source which the thermometer is measuring (for hot sources). The increase in signal with source diameter is shown in Figure 2. The SSE is checked annually as any contamination of optical components will increase this uncertainty.

\subsubsection{Non-linearity}

Silicon photodiodes are extremely linear, but a limit of $0.05 \%$ signal is placed on the linearity and annual checks are made to make sure that any non-linearity is within this limit. 


\subsubsection{Other contributions}

With decreasing impact on the overall uncertainty of the scale realisation the following contributions are allowed for: determination of reference photocurrent using the copper fixed-point cell, drift between the 6-monthly determination of the reference photocurrent, and switching between amplifier gains. The LP3 itself is temperature stabilised and is used in a laboratory controlled to $22 \pm 2{ }^{\circ} \mathrm{C}$, with due allowance in the uncertainty budget made for changes in filter temperature within this range.

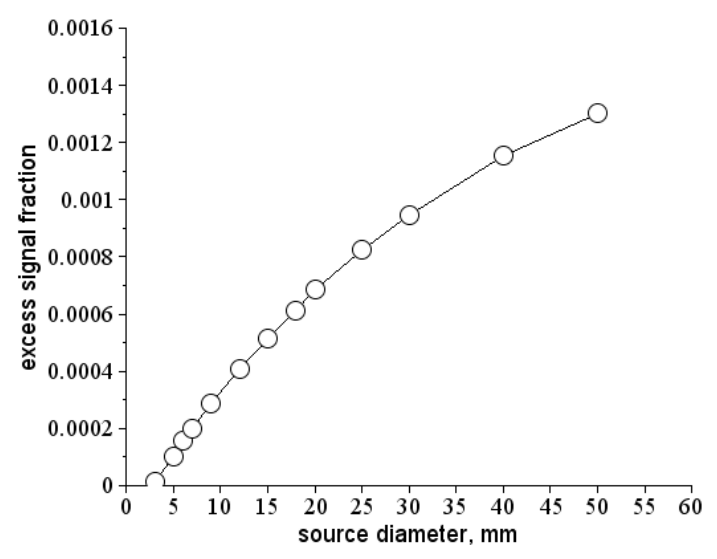

Figure 2. Size-of-source effect of LP3 thermometer showing excess signal fraction measured relative to a $3 \mathrm{~mm}$ diameter source.

\subsection{By use of high temperature fixed-point cells}

It was noted above that various time intensive assessments of the various key parameters for the LP3 have to be carried out at intervals ranging from 6-months to 2-years to enable the device to operate to the CMC uncertainties. This phasing allows for the possibility of arranging for all evaluations to coincide which means that the LP3 has, immediately after these assessments, its optimum low uncertainty performance. For example the drift components in the uncertainty budget are negligible immediately after calibration. At this time, immediately after determination of spectral response, the LP3 is used to assign temperatures to stable HTFP reference standards.

\subsubsection{High temperature fixed points}

The HTFP reference standards accepted for UKAS accredited calibration use are fixed-point cells of rhenium-carbon (nominally $2474{ }^{\circ} \mathrm{C}$ ), platinum-carbon $\left(1738{ }^{\circ} \mathrm{C}\right)$ and cobalt-carbon $\left(1324{ }^{\circ} \mathrm{C}\right)$. It has been established that the eutectic melting temperatures of these HTFPs are stable [9] and highly reproducible [10]. At NPL, their ITS-90 temperature can be determined with $k=2$ uncertainties of $0.018 \%$ for $\mathrm{Co}-\mathrm{C}, 0.023 \%$ for Pt-C and for $0.035 \%$ Re-C, where the uncertainty is in terms of percentage of degrees Celsius temperature. With the ITS-
90 temperatures of these fixed point cell determined these sources can be used as calibration references to directly realise a scale at the UKAS accredited level of $0.05 \%$ for a suitable customer thermometer. The fixed points are checked on an annual basis and a full recalibration is carried out every two years to coincide with the standard radiation thermometer wavelength calibration.

\subsubsection{Interpolated scale}

The ITS-90 above the silver point is based on extrapolation, but using three HTFPs with assigned ITS90 temperatures an interpolation based scheme, using the Sakuma-Hattori equation [11], relates the temperature $T$ to the signal $S$ with three adjustable parameters: $A, B$ and $C$ by

$$
S=\frac{C}{\exp \left(c_{2} /(A T+B)\right)-1}
$$

This equation is based on Planck's radiation law with the assumption that the wavelength $\lambda$ at a source radiance temperature $T$ can be modelled by $\lambda=A+B / T$, which is clearly related to the effective wavelength given by (2) above. The parameter $B$ arises from the finite bandwidth of the filter. It is also possible to determine $B$ by, for example, using manufacturers' data for filter and detector response, in a way that is reasonably insensitive on the temperature so determined, and in that case only two calibration points are needed.

\section{Calibration of customer thermometers}

The two approaches to scale realisation described in Sections 2.1 and 2.2 above are the basis for calibrating customer radiation thermometers at NPL.

In the first case, the calibrated LP3 is used to assign a temperature to a Thermogauge blackbody radiance source which acts as a transfer to assign a calibration to the customer instrument. Customer radiation thermometers with a field-of-view up to $12 \mathrm{~mm}\left(20 \mathrm{~mm}\right.$ to $\left.2500{ }^{\circ} \mathrm{C}\right)$ can be calibrated using this approach. The calibration uncertainty includes the calibration of the standard radiation thermometer, and the uniformity, stability and emissivity of the variable temperature blackbody source. Finally, the assessed uncertainty of the customer thermometer is added, with components for repeatability, resolution and SSE. If the device has previously been calibrated, the reason for any significant change in calibration (if detected) is investigated.

In the second case some combination of the primary copper point and calibrated HTFPs is used. Provided the spectral response is known to certain limits three HTFPs are used, though in principle two fixed points suffice [12]. All the fixed-point cells have apertures of $3 \mathrm{~mm}$ and so this method is limited to radiation thermometers with a field-of-view of $1.5 \mathrm{~mm}$ or less. Because of the small number of options for suitable thermometers NPL has 
developed a suitable device, designated the ATK [13], which can be supplied with a calibration based on this method. It has been shown that the ATK instrument is stable and robust enough to be transported and can be calibrated with an uncertainty substantially lower than can be achieved by calibration by comparison.

\section{Discussion}

In principle a radiation thermometer can leave NPL with a UKAS accredited calibration certificate that has uncertainties that are close to, or even at, the CMC value. In reality this usually does not happen, with the performance of the instrument being calibrated generally being the limiting factor. For most end-users a final uncertainty of $5-10{ }^{\circ} \mathrm{C}$ at $2000{ }^{\circ} \mathrm{C}$ suffices. However, there may be circumstances when lower uncertainties are required, for example where a radiation thermometer is used as a reference standard in a calibration laboratory. In this case the HTFP route can give benefit.

In particular a primary scale can be set up in minimal time with relatively simple equipment. A recent experiment demonstrated that a scale with primary level uncertainties could be established in three days at a calibration laboratory [14]. In the near future, the $M e P-K$ will specify HTFP melting temperatures and uncertainties. This route will then qualify as being a primary temperature scale (i.e. established in terms of the definition of the unit) and will greatly improve access to national standards with, potentially, calibration laboratories having their own set of HTFPs. In the meantime this route allows ITS-90 to be delivered at reduced cost and with greater confidence. It is also a means by which suitable radiation thermometers can be calibrated with much lower uncertainty. In effect, a relatively low cost instrument can be calibrated to an uncertainty that would usually be reserved for a top-level standard device.

\section{Conclusion}

The uncertainty in realising an ITS-90 temperature scale has remained largely unchanged since NPL calibration capability was reported in 1999 [15]. What has changed is the method by which the ITS-90 can be realised and disseminated. The conventional ITS-90 route relies on the stability and repeatability of equipment. It potentially offers the lowest uncertainties, but for reasons of practicality and cost the calibration steps are performed at intervals and so allowance has to be made for drifts. This increases the uncertainty of the realisation, and tends to be costly in terms of optimum realisation. It also requires significant outlay in capital equipment.

In contrast the use of HTFPs facilitates a realisation of the ITS-90 that relies on an essentially drift free process. The possibility is opened for secondary laboratories to have a direct realisation of a low uncertainty scale based on HTFPs with assigned temperatures, potentially negating the need for costly calibration of reference radiation thermometers at the NMI.

\section{References}

[1] Procès-Verbaux du Comité International des Poids et Mesures, 78th meeting, 1989, pp. T1-T21 (French version) and pp. T23-T42 (in English).

[2] G. Machin, Temperature: Its Measurement and Control in Science and Industry (AIP, Melville, New York), 8, 305 (2013)

[3] D. Lowe, Meas. Sci. Technol., 24, 015901 (2013)

[4] G. Machin, P. Bloembergen, K. Anhalt, J. Hartmann, M. Sadli, P. Saunders, E. Woolliams, Y. Yamada, H. Yoon, Int. J. Thermophys. 31, 1779-1788 (2010)

[5] B. Fellmuth, J. Fischer, G. Machin, S. Picard, P. Steur, O. Tamura, R. White, H. Yoon, "The kelvin redefinition and its mise en pratique", Phil. Trans R. Soc. A. (2015) submitted

[6] H. McEvoy, M. Sadli, F. Bourson, S. Briaudeau, B. Rougié, Metrologia 50, 559

[7] J. Fischer, G. Neuer, E. Schreiber, R. Thomas, Proc. Tempmeko2001 (VDE Verlag, Berlin), 801 (2002)

[8] E. Schreiber, G. Neuer Proc. Tempmeko04 (FSB/LPM, Zagreb) 527 (2004)

[9] M. Sadli, P. Bloembergen, B. Khlevnoy, T. Wang, Y. Yamada, G. Machin Int. J. Thermophys. 32, 1786 (2011)

[10] D. Lowe, Y. Yamada, Metrologia 43, S135 (2006)

[11] P. Saunders, Metrologia 34, 201 (2000)

[12] P. Bloembergen, Y. Yamada, Int. J. Thermophys. 32, 45 (2011)

[13] D. Lowe, M. Broughton, G. Machin, J. R. Wilmott, "Comparison of extrapolated and interpolated temperature scales from $1000{ }^{\circ} \mathrm{C}$ to $2500{ }^{\circ} \mathrm{C}$ between a national measurement institute and an ISO17025 accredited calibration laboratory" submitted Measurement 2015

[14] J. R. Willmott, D. Lowe, M. Broughton, G. Machin, these proceedings.

[15] G. Machin, proc. Metrologie 99, $9^{\text {th }}$ International Metrology Conference (Mouvement Francais pour la Qualite) 556 (1999) 